\title{
Faber Polynomial Coefficients of Classes of Meromorphic Bistarlike Functions
}

\author{
Jay M. Jahangiri ${ }^{1}$ and Samaneh G. Hamidi ${ }^{2}$ \\ ${ }^{1}$ Department of Mathematical Sciences, Kent State University, Burton, OH 44021-9500, USA \\ ${ }^{2}$ Department of Mathematics, Brigham Young University, Provo, UT 84602, USA
}

Correspondence should be addressed to Jay M. Jahangiri; jjahangi@kent.edu

Received 15 December 2014; Accepted 8 January 2015

Academic Editor: A. Zayed

Copyright (c) 2015 J. M. Jahangiri and S. G. Hamidi. This is an open access article distributed under the Creative Commons Attribution License, which permits unrestricted use, distribution, and reproduction in any medium, provided the original work is properly cited.

\begin{abstract}
Applying the Faber polynomial coefficient expansions to certain classes of meromorphic bistarlike functions, we demonstrate the unpredictability of their early coefficients and also obtain general coefficient estimates for such functions subject to a given gap series condition. Our results improve some of the coefficient bounds published earlier.
\end{abstract}

Let $\Sigma$ be the family of functions $g$ of the form

$$
g(z)=\frac{1}{z}+b_{0}+\sum_{n=1}^{\infty} b_{n} z^{n}
$$

that are univalent in the punctured unit disk $\mathbb{D}:=\{z: 0<$ $|z|<1\}$.

For the real constants $A$ and $B(0 \leq B \leq 1 ;-B \leq A<B)$, let $\Sigma(A, B)$ consist of functions $g \in \Sigma$ so that

$$
\frac{z g^{\prime}(z)}{g(z)}=-\frac{1+A \varphi(z)}{1+B \varphi(z)}
$$

where $\varphi(z)=\sum_{n=1}^{\infty} c_{n} z^{n}$ is a Schwarz function; that is, $\varphi(z)$ is analytic in the open unit disk $|z|<1$ and $|\varphi(z)| \leq|z|<1$. Note that $\left|c_{n}\right| \leq 1$ (Duren [1]) and the functions in $\Sigma(A, B)$ are meromorphic starlike in the punctured unit disk $\mathbb{D}$ (e.g., see Clunie [2] and Karunakaran [3]). It has been proved by Libera and Livingston [4] and Karunakaran [3] that $\left|b_{n}\right| \leq$ $(B-A) /(n+1)$ for $g \in \Sigma[A, B]$.
The coefficients of $h=g^{-1}$, the inverse map of $g$, are given by the Faber polynomial expansion (e.g., see Airault and Bouali [5] or Airault and Ren [6, page 349])

$$
\begin{aligned}
h(w) & =g^{-1}(w)=\frac{1}{w}+\sum_{n=0}^{\infty} B_{n} w^{n} \\
& =\frac{1}{w}-b_{0}-\sum_{n \geq 1} \frac{1}{n} K_{n+1}^{n}\left(b_{0}, b_{1}, \ldots, b_{n}\right) w^{n},
\end{aligned}
$$

where $w \in \mathbb{D}$,

$$
\begin{aligned}
K_{n+1}^{n} & \left(b_{0}, b_{1}, \ldots, b_{n}\right) \\
= & n b_{0}^{n-1} b_{1}+n(n-1) b_{0}^{n-2} b_{2} \\
& +\frac{1}{2} n(n-1)(n-2) b_{0}^{n-3}\left(b_{3}+b_{1}^{2}\right) \\
& +\frac{n(n-1)(n-2)(n-3)}{3 !} b_{0}^{n-4}\left(b_{4}+3 b_{1} b_{2}\right) \\
& +\sum_{j \geq 5} b_{0}^{n-j} V_{j},
\end{aligned}
$$

and $V_{j}$ is a homogeneous polynomial of degree $j$ in the variables $b_{1}, b_{2}, \ldots, b_{n}$. 
In 1923, Löwner [7] proved that the inverse of the Koebe function $k(z)=z /(1-z)^{2}$ provides the best upper bounds for the coefficients of the inverses of analytic univalent functions. Although the estimates for the coefficients of the inverses of analytic univalent functions have been obtained in a surprisingly straightforward way (e.g., see [8, page 104]), the case turns out to be a challenge when the biunivalency condition is imposed on these functions. A function is said to be biunivalent in a given domain if both the function and its inverse are univalent there. By the same token, a function is said to be bistarlike in a given domain if both the function and its inverse are starlike there. Finding bounds for the coefficients of classes of biunivalent functions dates back to 1967 (see Lewin [9]). The interest on the bounds for the coefficients of subclasses of biunivalent functions picked up by the publications [10-14] where the estimates for the first two coefficients of certain classes of biunivalent functions were provided. Not much is known about the higher coefficients of the subclasses biunivalent functions as Ali et al. [13] also declared that finding the bounds for $\left|a_{n}\right|$, $n \geq 4$, is an open problem. In this paper, we use the Faber polynomial expansions of the functions $g$ and $h=g^{-1}$ in $\Sigma(A, B)$ to obtain bounds for their general coefficients $\left|a_{n}\right|$ and provide estimates for the early coefficients of these types of functions.

We will need the following well-known two lemmas, the first of which can be found in [15] (also see Duren [1]).

Lemma 1. Let $p(z)=1+\sum_{n=1}^{\infty} p_{n} z^{n}$ be so that $\operatorname{Re}(p(z))>0$ for $|z|<1$. If $\alpha \geq-1 / 2$, then

$$
\left|p_{2}+\alpha p_{1}^{2}\right| \leq 2+\alpha\left|p_{1}\right|^{2}
$$

Consequently, we have the following lemma, in which we will provide a short proof for the sake of completeness.

Lemma 2. Consider the Schwarz function $\varphi(z)=\sum_{n=1}^{\infty} c_{n} z^{n}$, where $|\varphi(z)|<1$ for $|z|<1$. If $\gamma \geq 0$, then

$$
\left|c_{2}+\gamma c_{1}^{2}\right| \leq 1+(\gamma-1)\left|c_{1}\right|^{2} \text {. }
$$

Proof. Write

$$
p(z)=\frac{[1+\varphi(z)]}{[1-\varphi(z)]}
$$

where $p(z)=1+\sum_{n=1}^{\infty} p_{n} z^{n}$ is so that $\operatorname{Re}(p(z))>0$ for $|z|<1$. Comparing the corresponding coefficients of powers of $z$ in $p(z)=[1+\varphi(z)] /[1-\varphi(z)]$ shows that $p_{1}=2 c_{1}$ and $p_{2}=$ $2\left(c_{2}+c_{1}^{2}\right)$. obtain

By substituting for $p_{1}=2 c_{1}$ and $p_{2}=2\left(c_{2}+c_{1}^{2}\right)$ in (5), we

$$
\left|2\left(c_{2}+c_{1}^{2}\right)+\alpha\left(2 c_{1}\right)^{2}\right| \leq 2+\alpha\left|2 c_{1}\right|^{2}
$$

or

$$
\left|c_{2}+(1+2 \alpha) c_{1}^{2}\right| \leq 1+2 \alpha\left|c_{1}\right|^{2}
$$

Now, (6) follows upon substitution of $\gamma=1+2 \alpha \geq 0$ in the above inequality.

In the following theorem, we will observe the unpredictability of the early coefficients of the functions $g$ and its inverse map $h=g^{-1}$ in $\Sigma[A, B]$, providing an estimate for the general coefficients of such functions.

Theorem 3. For $0 \leq B \leq 1$ and $-B \leq A<B$, let the function $g$ and its inverse map $h=g^{-1}$ be in $\Sigma[A, B]$. Then,

(i) $\left|b_{0}\right| \leq \begin{cases}\frac{B-A}{\sqrt{2 B-A}}, & \text { if } 2 B-A \geq 1 ; \\ B-A, & \text { otherwise, }\end{cases}$

(ii) $\left|b_{1}\right|$

$$
\leq \begin{cases}\frac{B-A}{2}-\frac{1-A-2 B}{2(B-A)}\left|b_{0}\right|^{2}, & \text { if } 0 \leq A \leq 1-2 B \\ \frac{B-A}{2}, & \text { otherwise, }\end{cases}
$$

(iii) $\left|b_{1} \pm \frac{2 B-A}{2(B-A)} b_{0}^{2}\right| \leq \frac{B-A}{2}$,

(iv) $\left|b_{n}\right| \leq \frac{B-A}{n+1}$; if $b_{k}=0$ for $0 \leq k \leq n-1$.

Proof. Consider the function $g \in \sum$ given by (1). Therefore (see $[5,6])$

$$
\frac{z g^{\prime}(z)}{g(z)}=-1-\sum_{n=0}^{\infty} F_{n+1}\left(b_{0}, b_{1}, b_{2}, \ldots, b_{n}\right) z^{n+1}
$$

where $F_{n+1}\left(b_{0}, b_{1}, b_{2}, \ldots, b_{n}\right)$ is a Faber polynomial of degree $n+1$. We note that $F_{1}=-b_{0}, F_{2}=b_{0}^{2}-2 b_{1}, F_{3}=-b_{0}^{3}+$ $3 b_{1} b_{0}-3 b_{2}, F_{4}=b_{0}^{4}-4 b_{0}^{2} b_{1}+4 b_{0} b_{2}+2 b_{1}^{2}-4 b_{3}$, and $F_{5}=$ $-b_{0}^{5}+5 b_{0}^{3} b_{1}-5 b_{0}^{2} b_{2}-5 b_{0} b_{1}^{2}+5 b_{1} b_{2}+5 b_{0} b_{3}-5 b_{4}$. In general (Bouali [16, page 52]),

$$
\begin{aligned}
& F_{n+1}\left(b_{0}, b_{1}, \ldots, b_{n}\right) \\
& \quad=\sum_{i_{1}+2 i_{2}+\cdots+(n+1) i_{n+1}=n+1} A\left(i_{1}, i_{2}, \ldots, i_{n+1}\right) b_{0}^{i_{1}} b_{1}^{i_{2}} \ldots b_{n}^{i_{n+1}},
\end{aligned}
$$

where

$$
\begin{aligned}
& A\left(i_{1}, i_{2}, \ldots, i_{n+1}\right) \\
& \quad:=(-1)^{(n+1)+2 i_{1}+\cdots+(n+2) i_{n+1}} \frac{\left(i_{1}+i_{2}+\cdots+i_{n+1}-1\right) !(n+1)}{\left(i_{1} !\right)\left(i_{2} !\right) \cdots\left(i_{n+1} !\right)} .
\end{aligned}
$$


Similarly, for the inverse map $h=g^{-1}$, we have

$$
\frac{w h^{\prime}(w)}{h(w)}=-1-\sum_{n=1}^{\infty} F_{n+1}\left(B_{0}, B_{1}, B_{2}, \ldots, B_{n}\right) w^{n+1}
$$

where $F_{n+1}\left(B_{0}, B_{1}, B_{2}, \ldots, B_{n}\right)$ is a Faber polynomial of degree $n+1$ given by

$$
\begin{aligned}
F_{n+1}= & -\frac{n(n-(n+1)) !}{n !(n-2 n) !} B_{0}^{n} \\
& -\frac{n(n-(n+1)) !}{(n-2) !(n-(2 n-1)) !} B_{0}^{n-2} B_{1} \\
& -\frac{n(n-(n+1)) !}{(n-3) !(n-(2 n-2)) !} B_{0}^{n-3} B_{2} \\
& -\frac{n(n-(n+1)) !}{(n-4) !(n-(2 n-3)) !} B_{0}^{n-4} \\
& \cdot\left(B_{3}+\frac{n-(2 n-3)}{2} B_{1}^{2}\right)-\sum_{j \geq 5} B_{0}^{n-j} K_{j},
\end{aligned}
$$

where $K_{j}$ is a homogeneous polynomial of degree $j$ in the variables $B_{1}, B_{2}, \ldots, B_{n-1}$ and

$$
B_{0}=-b_{0}, \quad B_{n}=-\frac{1}{n} K_{n+1}^{n}\left(b_{0}, b_{1}, \ldots, b_{n}\right) .
$$

Since both $g$ and its inverse map $h=g^{-1}$ are in $\Sigma[A, B]$, the Faber polynomial expansion yields (also see Duren [1, pages 118-119])

$$
\begin{aligned}
\frac{z g^{\prime}(z)}{g(z)} & =-\frac{1+A \varphi(z)}{1+B \varphi(z)} \\
& =-1+\sum_{n=1}^{\infty}(A-B) K_{n}^{-1}\left(c_{1}, c_{2}, \ldots, c_{n}, B\right) z^{n}, \\
\frac{w h^{\prime}(w)}{h(w)} & =-\frac{1+A \psi(w)}{1+B \psi(w)} \\
& =-1+\sum_{n=1}^{\infty}(A-B) K_{n}^{-1}\left(d_{1}, d_{2}, \ldots, d_{n}, B\right) w^{n},
\end{aligned}
$$

where $\varphi(z)=\sum_{n=1}^{\infty} c_{n} z^{n}$ and $\psi(w)=\sum_{n=1}^{\infty} d_{n} w^{n}$ are two Schwarz functions; that is, $|\varphi(z)|<1$ for $|z|<1$ and $|\psi(w)|<$ 1 for $|w|<1$.
In general (see Airault [17] or Airault and Bouali [5]), the coefficients $K_{n}^{p}\left(k_{1}, k_{2}, \ldots, k_{n}, B\right)$ are given by

$$
\begin{aligned}
K_{n}^{p}( & \left.k_{1}, k_{2}, \ldots, k_{n}, B\right) \\
= & \frac{p !}{(p-n) ! n !} k_{1}^{n} B^{n-1} \\
& +\frac{p !}{(p-n+1) !(n-2) !} k_{1}^{n-2} k_{2} B^{n-2} \\
& +\frac{p !}{(p-n+2) !(n-3) !} k_{1}^{n-3} k_{3} B^{n-3} \\
& +\frac{p !}{(p-n+3) !(n-4) !} k_{1}^{n-4} \\
& \cdot\left[k_{4} B^{n-4}+\frac{p-n+3}{2} k_{2}^{2} B\right] \\
& +\frac{p !}{(p-n+4) !(n-5) !} k_{1}^{n-5} \\
& \cdot\left[k_{5} B^{n-5}+(p-n+4) k_{2} k_{3} B\right] \\
& +\sum_{j \geq 6} k_{1}^{n-j} X_{j},
\end{aligned}
$$

where $X_{j}$ is a homogeneous polynomial of degree $j$ in the variables $k_{2}, k_{3}, \ldots, k_{n}$.

Comparing the corresponding coefficients of (11) and (17) implies

$$
-F_{n+1}\left(b_{0}, b_{1}, b_{2}, \ldots, b_{n}\right)=(A-B) K_{n+1}^{-1}\left(c_{1}, c_{2}, \ldots, c_{n+1}, B\right) \text {. }
$$

Similarly, comparing the corresponding coefficients of (14) and (18) gives

$$
\begin{aligned}
- & F_{n+1}\left(B_{0}, B_{1}, B_{2}, \ldots, B_{n}\right) \\
& =(A-B) K_{n+1}^{-1}\left(d_{1}, d_{2}, \ldots, d_{n+1}, B\right) .
\end{aligned}
$$

Substituting $n=0, n=1$, and $n=2$ in (16), (20), and (21), respectively, yields

$$
\begin{gathered}
b_{0}=(A-B) c_{1}, \\
-b_{0}=(A-B) d_{1}, \\
2 b_{1}-b_{0}^{2}=(B-A)\left(c_{1}^{2} B-c_{2}\right), \\
2 b_{1}+b_{0}^{2}=(A-B)\left(d_{1}^{2} B-d_{2}\right) .
\end{gathered}
$$

Taking the absolute values of either equation in (22), we obtain $\left|b_{0}\right| \leq B-A$. Obviously, from (22), we note that $c_{1}=-d_{1}$. Solving the equations in (23) for $b_{0}^{2}$ and then adding them gives

$$
2 b_{0}^{2}=(B-A)\left(c_{2}+d_{2}-B c_{1}^{2}-B d_{1}^{2}\right) \text {. }
$$


Now, in light of (22), we conclude that

$$
2 b_{0}^{2}=(B-A)\left(c_{2}+d_{2}-\frac{2 B}{(B-A)^{2}} b_{0}^{2}\right) .
$$

Once again, solving for $b_{0}^{2}$ and taking the square root of both sides, we obtain

$$
\left|b_{0}\right| \leq \sqrt{\frac{(B-A)^{2}\left(c_{2}+d_{2}\right)}{2(2 B-A)}} \leq \frac{B-A}{\sqrt{2 B-A}} .
$$

Now, the first part of Theorem 3 follows since for $2 B-A>1$ it is easy to see that

$$
\frac{B-A}{\sqrt{2 B-A}}<B-A .
$$

Adding the equations in (23) and using the fact that $c_{1}=-d_{1}$, we obtain

$$
4 b_{1}=(B-A)\left[\left(d_{2}-c_{2}\right)-\left(d_{1}^{2}-c_{1}^{2}\right) B\right]=(B-A)\left(d_{2}-c_{2}\right) .
$$

Dividing by 4 and taking the absolute values of both sides yield

$$
\left|b_{1}\right| \leq \frac{B-A}{2} .
$$

On the other hand, from the second equations in (22) and (23), we obtain

$$
2 b_{1}=(B-A)\left(d_{2}+A d_{1}^{2}-2 B d_{1}^{2}\right) .
$$

Taking the absolute values of both sides and applying Lemma 2, it follows that

$$
\begin{aligned}
\left|b_{1}\right| & \leq \frac{1}{2}(B-A)\left(\left|d_{2}+A d_{1}^{2}\right|+2 B\left|d_{1}\right|^{2}\right) \\
& \leq \frac{1}{2}(B-A)\left(1+(A-1)\left|d_{1}\right|^{2}+2 B\left|d_{1}\right|^{2}\right) \\
& =\frac{B-A}{2}-\frac{1-A-2 B}{2(B-A)}\left|b_{0}\right|^{2} .
\end{aligned}
$$

This concludes the second part of Theorem 3 since for $0<$ $A<1-2 B$ we have

$$
\frac{B-A}{2}-\frac{1-A-2 B}{2(B-A)}\left|b_{0}\right|^{2}<\frac{B-A}{2} .
$$

Substituting (22) in (23), we obtain

$$
\begin{aligned}
& 2 b_{1}-b_{0}^{2}=(B-A)\left(\frac{B}{(B-A)^{2}} b_{0}^{2}-c_{2}\right), \\
& 2 b_{1}+b_{0}^{2}=(A-B)\left(\frac{B}{(A-B)^{2}} b_{0}^{2}-d_{2}\right) .
\end{aligned}
$$

Following a simple algebraic manipulation, we obtain the coefficient body

$$
\left|b_{1} \pm \frac{2 B-A}{2(B-A)} b_{0}^{2}\right| \leq \frac{B-A}{2} .
$$

Finally, for $b_{k}=0,0 \leq k \leq n-1,(20)$ yields

$$
(n+1) b_{n}=(A-B) c_{n+1} \text {. }
$$

Solving for $b_{n}$ and taking the absolute values of both sides, we obtain

$$
\left|b_{n}\right| \leq \frac{B-A}{n+1}
$$

Remark 4. The estimate $\left|b_{0}\right| \leq(B-A) / \sqrt{2 B-A}$ given by Theorem 3(i) is better than that given in ([14, Theorem 2(i) $]$ ).

Remark 5. In ([3, Theorem 1$])$, the bound $\left|b_{n}\right| \leq(B-A) /(n+1)$ was declared to be sharp for the coefficients of the function $g \in \Sigma[A, B]$. The coefficient estimates $\left|b_{0}\right| \leq(B-A) / \sqrt{2 B-A}$ and $\left|b_{1}\right| \leq(B-A) / 2-((1-A-2 B) / 2(B-A))\left|b_{0}\right|^{2}$ given by Theorem 3 show that the coefficient bound $\left|b_{n}\right| \leq(B-A) /(n+$ $1)$ is not sharp for the meromorphic bistarlike functions, that is, if both $g$ and its inverse map $g^{-1}$ are in $\Sigma[A, B]$. Finding sharp coefficient bound for meromorphic bistarlike functions remains an open problem.

\section{Conflict of Interests}

The authors declare that there is no conflict of interests regarding the publication of this paper.

\section{References}

[1] P. L. Duren, Univalent Functions, vol. 259 of Grundlehren der Mathematischen Wissenschaften, Springer, New York, NY, USA, 1983.

[2] J. Clunie, "On meromorphic schlicht functions," Journal of the London Mathematical Society, vol. 34, pp. 215-216, 1959.

[3] V. Karunakaran, "On a class of meromorphic starlike functions in the unit disc," Mathematical Chronicle, vol. 4, no. 2-3, pp. 112121, 1976.

[4] R. J. Libera and A. E. Livingston, "Bounded functions with positive real part," Czechoslovak Mathematical Journal, vol. 22, pp. 195-209, 1972.

[5] H. Airault and A. Bouali, "Differential calculus on the Faber polynomials," Bulletin des Sciences Mathématiques, vol. 130, no. 3, pp. 179-222, 2006.

[6] H. Airault and J. Ren, "An algebra of differential operators and generating functions on the set of univalent functions," Bulletin des Sciences Mathématiques, vol. 126, no. 5, pp. 343-367, 2002.

[7] K. Löwner, "Untersuchungen über schlichte konforme Abbildungen des Einheitskreises. I," Mathematische Annalen, vol. 89, no. 1-2, pp. 103-121, 1923.

[8] J. G. Krzyz, R. J. Libera, and E. Złotkiewicz, "Coefficients of inverses of regular starlike functions," Annales Universitatis Mariae Curie-Skłodowska A, vol. 33, pp. 103-110, 1979.

[9] M. Lewin, "On a coefficient problem for bi-univalent functions," Proceedings of the American Mathematical Society, vol. 18, no. 1, pp. 63-63, 1967.

[10] D. A. Brannan and T. S. Taha, "On some classes of biunivalent functions," Studia Universitatis Babeş-Bolyai Series Mathematica, vol. 31, no. 2, pp. 70-77, 1986. 
[11] H. M. Srivastava, A. K. Mishra, and P. Gochhayat, "Certain subclasses of analytic and bi-univalent functions," Applied Mathematics Letters, vol. 23, no. 10, pp. 1188-1192, 2010.

[12] B. A. Frasin and M. K. Aouf, "New subclasses of bi-univalent functions," Applied Mathematics Letters, vol. 24, no. 9, pp. 15691573, 2011.

[13] R. M. Ali, S. K. Lee, V. Ravichandran, and S. Supramaniam, "Coefficient estimates for bi-univalent Ma-Minda starlike and convex functions," Applied Mathematics Letters, vol. 25, no. 3, pp. 344-351, 2012.

[14] S. G. Hamidi, S. A. Halim, and J. M. Jahangiri, "Faber polynomial coefficient estimates for meromorphic bi-starlike functions," International Journal of Mathematics and Mathematical Sciences, vol. 2013, Article ID 498159, 4 pages, 2013.

[15] M. Jahangiri, "On the coefficients of powers of a class of Bazilevic functions," Indian Journal of Pure and Applied Mathematics, vol. 17, no. 9, pp. 1140-1144, 1986.

[16] A. Bouali, "Faber polynomials, Cayley-Hamilton equation and Newton symmetric functions," Bulletin des Sciences Mathématiques, vol. 130, no. 1, pp. 49-70, 2006.

[17] H. Airault, "Remarks on Faber polynomials," International Mathematical Forum, vol. 3, no. 9-12, pp. 449-456, 2008. 


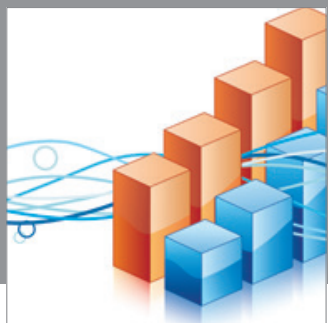

Advances in

Operations Research

mansans

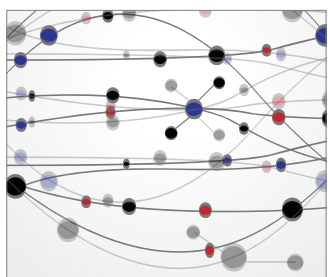

The Scientific World Journal
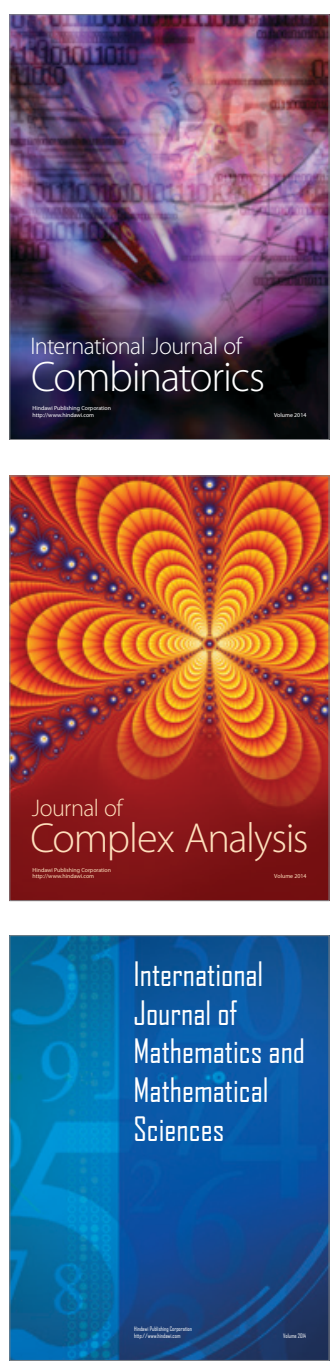
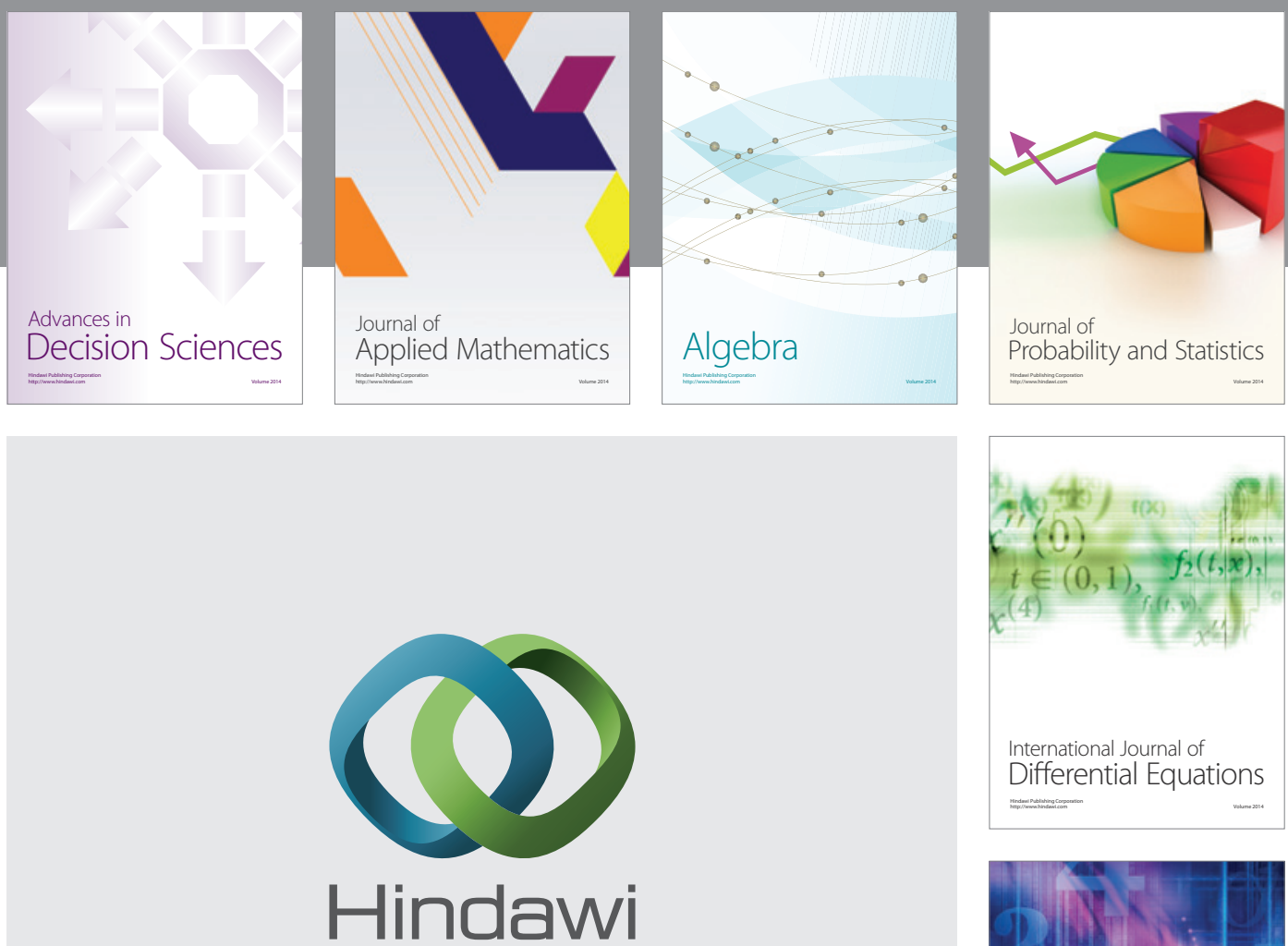

Submit your manuscripts at http://www.hindawi.com
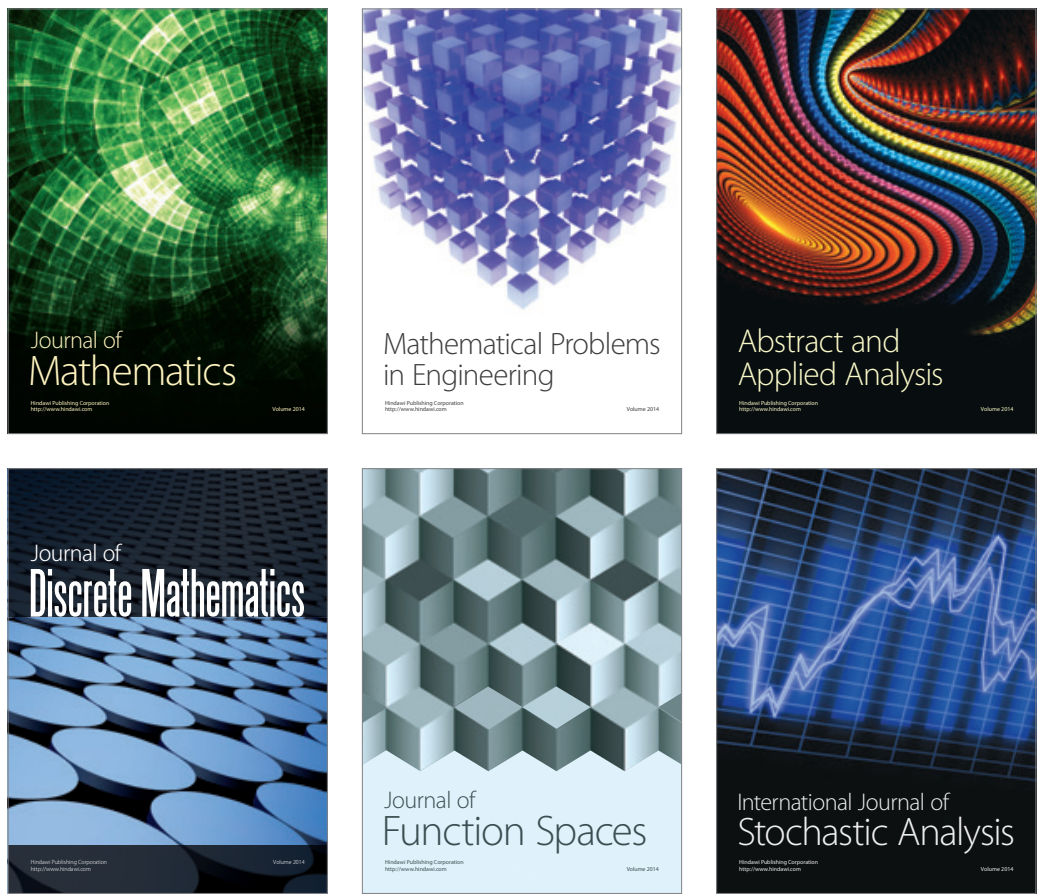

Journal of

Function Spaces

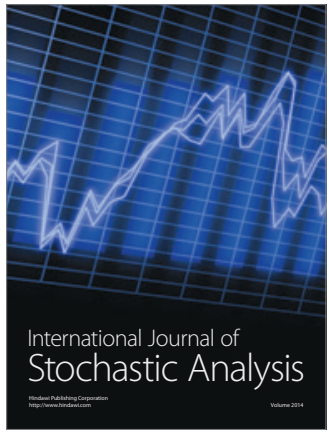

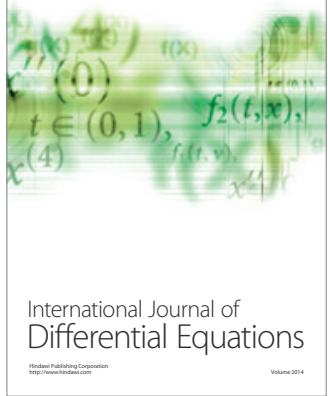
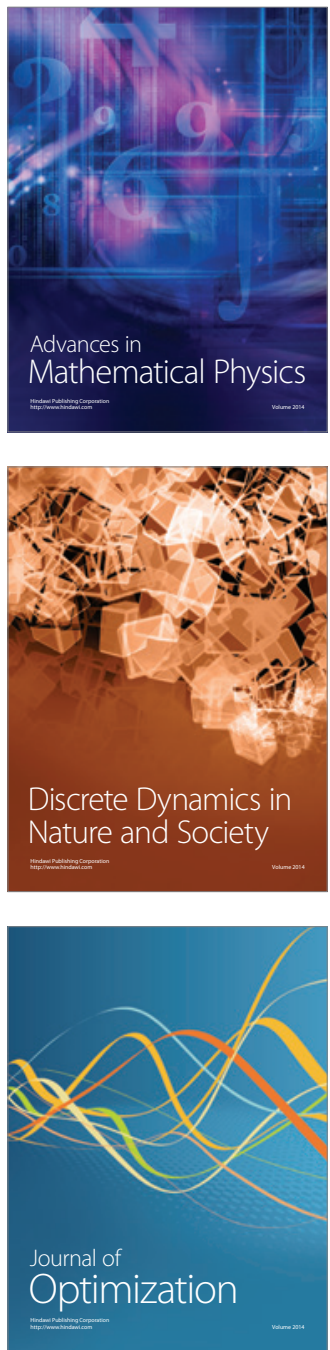